\title{
Pedagogía social
}

Social education / Pedagogia social

Compromiso de las universidades colombianas con la sustentabilidad

Colombian Universities' Commitment to Sustainability

Comprometimento das universidades colombianas com a sustentabilidade

Ángela María Plata Rangel, María Teresa Holguín Aguirre, Orlando Sáenz Zapata, William Manuel Mora Penagos, María Mercedes Callejas Restrepo

\section{Diagnóstico de sensibilidad medioambiental en estudiantes} universitarios

Diagnosing Environmental Awareness in University Students

Diagnóstico de sensibilidade meio ambiental em estudantes universitários

M. Eugenia Mediavilla, Silvia Medina Quintana, Ignacio González López 



\section{Compromiso de las universidades colombianas con la sustentabilidad}

Ángela María Plata Rangel

https://orcid.org/oooo-0002-1276-0926 Universidad Sergio Arboleda, Colombia angela.plata@usa.edu.co

\section{Orlando Sáenz Zapata}

https://orcid.org/000o-0003-1229-802X Universidad de Ciencias Aplicadas y Ambientales, Colombia osaenz@udca.edu.co

\section{María Mercedes Callejas Restrepo}

https://orcid.org/00oo-0003-4018-7079 Universidad de Ciencias Aplicadas y Ambientales, Colombia mcallejas@udca.edu.co
María Teresa Holguín Aguirre

https://orcid.org/o0oo-0002-9352-9223 Universidad Libre, Colombia mariat.holguina@unilibre.edu.co

William Manuel Mora Penagos

https://orcid.org/00oo-0003-4289-4842 Universidad Distrital Francisco José de Caldas, Colombia wmmorap@correo.udistrital.edu.co

\section{Resumen}

Este artículo presenta el nivel de institucionalización del compromiso con la sustentabilidad de las universidades colombianas. El estudio aborda cinco ámbitos institucionales: gobierno y participación; docencia y formación ambiental; investigación ambiental; extensión o proyección social ambiental; y gestión y ordenamiento ambiental. Se realizó mediante la aplicación de una encuesta a 60 instituciones de educación superior de Colombia. Para el análisis se utilizaron los indicadores de la estadistica descriptiva, cuyos resultados muestran que las universidades colombianas han avanzado en el compromiso con la sustentabilidad, pero aún se encuentran en un nivel bajo de la escala utilizada en la investigación. Solo el ámbito de docencia y formación ambiental se encuentra en un rango medio.

\section{Palabras clave (Fuente: tesauro de la Unesco)}

Colombia; desarrollo sostenible; educación superior; enseñanza superior; gestión educacional; gestión universitaria; universidades.

Recepción: 22/10/2019 | Envío a pares: 05/03/2020 | Aceptación por pares: 20/03/2020 | Aprobación: 06/04/2020 


\title{
Colombian Universities' Commitment to Sustainability
}

\begin{abstract}
This article presents the level of institutionalization of Colombian universities'commitment to sustainability. The study addresses five institutional areas: governance and participation, environmental teaching and training, environmental research, environmental and social outreach, and environmental management and planning. It is carried out by administering a survey to 60 higher education institutions in Colombia. For the analysis of results, descriptive statistic indicators are used. Results show that Colombian universities have progressed in their commitment to sustainability, but are still at a low level on the scale used in the research. Only the environmental teaching and training area has reached a medium level.
\end{abstract}

\section{Keywords (Source: Unesco thesaurus)}

Colombia; sustainable development; higher education; educational management; university management; universities. 


\section{Comprometimento das universidades colombianas com a sustentabilidade}

Resumo

Este artigo apresenta o nível de institucionalização do comprometimento com a sustentabilidade das universidades colombianas. O estudo aborda cinco âmbitos institucionais: governo e participação; docência e formação ambiental; pesquisa ambiental; extensão ou projeção social ambiental e gestão e ordenamento ambiental. Além disso, foi realizado mediante a aplicação de um questionário a 60 instituições de ensino superior da Colômbia. Para a análise de resultados, foram utilizados os indicadores da estatística descritiva. Os resultados mostram que as universidades colombianas vêm avançando no engajamento com a sustentabilidade, mas ainda se encontram em um nível baixo da escala utilizada na pesquisa. Somente o âmbito de docência e formação ambiental se encontra na média.

\section{Palavras-chave (Fonte: tesauro da Unesco)}

Colômbia; Desenvolvimento sustentável; ensino superior; educação superior; gestão educacional; gestão universitária; universidades. 
Los efectos negativos de la actividad humana sobre el ambiente transitan hacia la construcción de sociedades más sostenibles, lo que se ve reflejado, por ejemplo, en el interés de los gobiernos en dar respuesta a los Objetivos del Desarrollo Sostenible. Tal como lo resaltan Amérigo et al., "el análisis transculturaly la evolución de la crisis medioambiental están revelando nuevos planteamientos que ponen de manifiesto que la cuestión medioambiental está representada en la sociedad contemporánea por múltiples realidades que van más allá de una conceptualización dual u opuesta" (2017, p. 2). Es importante entonces analizar el papel que juegan las universidades frente a dichos planteamientos, pues es con fundamento en su responsabilidad y la integración de sus funciones sustantivas como contribuyen a la capacitación de las comunidades en las regiones. Por tanto, para ser coherentes con lo que promulgan, las universidades deben lograr que sus prácticas sean sustentables en su gestión tanto académica como administrativa, con el desafío de evaluar permanentemente cómo están respondiendo a esta integración (Alonso et al., 2015; Lozano, 2006; Wals, 2014).

Sin embargo, cada vez se reportan más dificultades asociadas al proceso evaluativo, entre ellas, las derivadas de la utilización de herramientas ajenas al contexto universitario. Otra dificultad es que las herramientas hacen más énfasis en el ámbito de la gestión ambiental (agua, energía, residuos) y es menos frecuente un enfoque holístico (Koester et al., 2006; Sterling, 2004) que abarque los ámbitos atendiendo a la extensión y proyección social, la docencia, la investigación, así como a las operaciones en el campus (Ceulemans et al., 2015; Fonseca et al., 2011; Lukman y Glavic, 2006). Adicionalmente, durante el año 2014, en el XIX Foro de Ministros de Ambiente de América Latina y el Caribe, se convocó a "desarrollar un diagnóstico por país sobre la inclusión de consideraciones ambientales en las universidades" (PNUMA, 2014), lo cual llevó al equipo de investigación Universidad, Ambiente y Sustentabilidad (UAS) a realizar un estudio sobre el nivel de institucionalización del compromiso con la sustentabilidad, en una muestra de 36 universidades colombianas, es decir, un 43,9\% del total de las 82 que hay en el país (Sáenz et al., 2017).

El objetivo de la investigación presentada en este artículo es reconocer el compromiso con la sustentabilidad por parte de un grupo de universidades colombianas, comparando los resultados de dos fases de estudio, a partir de la aplicación de una encuesta de 25 preguntas básicas. Para su desarrollo, se establecieron las diferencias entre los resultados globales de las dos fases. La primera contempló solo las respuestas Sí/No y en la segunda se realizó una verificación de la información. Las preguntas fueron tomadas y adaptadas del proyecto Red de Indicadores de Sostenibilidad Universitaria (RISU), titulado "Definición de indicadores para la evaluación de las políticas de sustentabilidad en universidades latinoamericanas", el cual buscaba definir un marco de análisis para la evaluación de las políticas de sostenibilidad en las universidades de la región (Benayas et al., 2014).

Al finalizar el análisis de los resultados, a partir de preguntas cerradas dicotómicas (Sí o NO), la investigación dio cuenta de un alto nivel de compromiso institucional de las universidades colombianas (Sáenz et al., 2017). Sin embargo, en una segunda fase se corroboraron los resultados obtenidos, analizando la información adicional aportada por cada universidad, a partir de tres criterios de verificación: ampliación de la información, aprobación oficial y divulgación en sus páginas web, donde se demuestra una variación importante de los resultados iniciales.

\section{Marco conceptual}

\section{Los sistemas ambientales universitarios: una estrategia integral para incorporar los temas de ambiente y sustentabilidad}

Abordar la inclusión de los temas de ambiente y sustentabilidad en las universidades requiere de una perspectiva sistémica, para lo cual es necesario adentrarnos en todos y cada uno de los procesos, funciones, políticas y objetivos de la educación superior, en la búsqueda de la coherencia académica 
y administrativa, a fin de comprometerse con una educación integral donde se impulse el trabajo interdisciplinario, transdisciplinario y transversal a los programas académicos y a los procesos de la gestión educativa.

La institucionalización del compromiso con la sustentabilidad en las instituciones de educación superior (IES) constituye un llamado a la responsabilidad social universitaria, en cuanto estas deben formar profesionales que prioricen la conservación del ambiente, la calidad de vida, la equidad social, entre otros. Como lo señalan González et al.:

La cultura de la sustentabilidad requiere, en definitiva, un cambio de modelo que permee a todos los estamentos de la comunidad universitaria y modifique substancialmente su forma de ser y de estar en ella, desde las esferas académica y profesional, ligadas a sus roles como docentes, investigadores, estudiantes o personal de gestión, incluso en los ámbitos deportivo, artístico, recreativo, de promoción cultural $y$ de reflexión crítica y politica, hasta la esfera personal y social, ligada a sus roles como ciudadanos. (2015, p. 87)

En lo que respecta a la perspectiva de sistemas y, en particular, a los sistemas sociales, Niklas Luhmann (1990) los identifica como complejos, autopoiéticos, con propiedades emergentes, respecto de los cuales siempre se debe identificar su composición, su estructura, sus mecanismos y su entorno.

Se puede afirmar entonces que las IES son sistemas sociales complejos, como también los campos del saber que estas abordan, entre ellos el ambiental. Por lo tanto, es posible hablar de Sistemas Ambientales Institucionales (SAI) que se organizan como un macrosistema y guardan relaciones con otros sistemas del contexto universitario.

Como estrategia para abordar los temas de ambiente y sustentabilidad en las Universidades, los SAI buscan la cohesión e integración de las fun- ciones sustantivas de la universidad con la gestión administrativa, con el fin de formar profesionales integrales que respondan a las necesidades del contexto, con competencias para el análisis y la búsqueda de soluciones frente a las problemáticas socioambientales.

El SAI no solo se relaciona internamente con otros sistemas de la misma universidad, sino que externamente guarda relaciones con otros sistemas de mayor jerarquía, como el Sistema Nacional Ambiental (SINA) y las redes universitarias como la Red Ambiental de Universidades Sostenibles y la Red Colombiana de Formación Ambiental. La Red RISU para Latinoamérica, o la Global Universities Partnership on Environment for Sustainability (GUPES o Alianza Mundial de Universidades por el Ambiente y la Sustentabilidad) (Mora, 2015).

La formación, en el contexto de la IES, debe propender por el desarrollo humano sustentable, a partir de procesos de análisis crítico enfocados en la generación de una cultura ciudadana que vincule aspectos conceptuales, técnicos, investigativos y participativos. Asimismo, dicha formación debe guardar coherencia con los diversos escenarios de la acción universitaria: docencia, investigación, extensión y proyección social, gestión administrativa, reflejando así una conexión entre el discurso y la práctica educativa.

Por otra parte, Holguín resalta que:

Una gestión educativa en temas de ambiente y sustentabilidad, concebida de manera sistémica, permite reconocer la sinergia que se debe dar permanentemente entre la academia y la administración, asi como el papel desde cada uno de los roles laborales y perfiles profesionales, en la comprensión de los problemas ambientales. (2017, p. 43)

La inclusión de los temas de ambiente y sustentabilidad en la educación superior debe propiciar el desarrollo de conocimientos y habilidades 
para que los profesionales establezcan relaciones sustentables con su entorno; además, como lo señala Holguín, se requiere

... de la participación de diversos saberes, puntos de vista y perspectivas, lo cual implica un trabajo de permanente de diálogo, análisis y sintesis, entre las diferentes facultades, los programas académicos, la administración, los directivos, los docentes, los estudiantes, los administrativos, el personal de servicios generales, la comunidad educativa en general, e, incluso, los vecinos, los proveedores, contratistas y todos aquellos que prestan servicios de diferente indole. (2017, p. 43)

Por su parte, la Política Nacional de Educación Ambiental (PNEA) señala que es necesario la inclusión de la dimensión ambiental en el currículo de la educación superior, tanto en los programas de formación inicial y de especialización de profesionales, como en el desarrollo de proyectos de investigación y en el servicio social (Ministerio de Educación Nacional y Ministerio de Ambiente de Colombia, 2002).

A mediados de la década del 2000, se empezó a hablar en Colombia de los Proyectos Ambientales Universitarios (PRAUS). Algunas instituciones los entendieron como una estrategia para la inclusión de la dimensión ambiental en el currículo y otras como una herramienta equivalente a los Proyectos Ambientales Escolares (PRAEs) que se tienen en los niveles de la educación primaria y secundaria. Sin embargo, no se logró su apropiación por parte de las universidades.

El presente estudio retoma la propuesta de Sistemas Ambientales Universitarios (SAU) formulada colectivamente en un proyecto desarrollado por el convenio de cooperación entre la Secretaría Distrital de Ambiente de Bogotá y la Universidad Piloto de Colombia, durante los años 2007 y 2008, titulado "Convenio de cooperación número 035-07 para desarrollar estrategias de investigación y gestión para la inclusión y dinamización de la dimensión ambiental en la Educación Superior en la ciudad de Bogotá" (Román, 2016).
Este trabajo dio paso a un libro titulado Sistema Ambiental Universitario. Modelo integrado para la inclusión de la dimensión ambiental y urbana en la educación superior, en el cual se define el Sistema Ambiental Universitario como un "conjunto de lineamientos, normas, orientaciones, planes, programas proyectos, actividades, metas e indicadores, así como recursos (humanos, técnicos y financieros) y responsables que orientan, regulan e interactúan en la planeación y gestión de la Universidad" (Román, 2016).

El Equipo UAS, por su parte, habla de Sistemas Ambientales Institucionales, para ampliar su cobertura a todas las IES, y lo define como

... el nivel más complejo de organización al cual una IES debe llegar en los temas de ambiente y sustentabilidad, donde la responsabilidad socioambiental es el contexto que mueve el desarro170 de todos los proyectos y acciones que deben emprender las IES para fortalecer la formación integral de los ciudadanos y reducir los impactos que puedan generar sobre el ambiente y la sociedad. (Universidad Libre, 2016)

La responsabilidad socioambiental es el eje y el marco general para la inclusión de los temas de ambiente y sustentabilidad en las IES. Parte de un concepto general de responsabilidad social, adaptado a las funciones y procesos de la universidad, según el cual se "busca responder de forma transparente y congruente a los intereses de las partes interesadas minimizando los impactos negativos y maximizando los positivos" (Vallaeys et al., 2009). Basados en lo anterior, el Equipo UAS establece los ámbitos para agrupar discursos y prácticas de ambiente y sustentabilidad desde una mirada sistémica. Estos son:

1. GobieRno y PARTICIPACIÓn: Hace referencia a la forma en que una IES se organiza para la toma de decisiones frente a los temas relacionados con ambiente y sustentabilidad, lo cual debe reflejar la institucionalización del compromi- 
so ambiental, consolidado, por ejemplo, en una política ambiental, una oficina responsable de la "ambientalización" administrativa y curricular, un presupuesto exclusivo, entre otros requerimientos.

2. DOCENCIA Y FORMACIÓN AMBIENTAL: Implica acciones concretas para que las IES incorporen en sus procesos de formación y docencia el componente de sustentabilidad ambiental. Hace referencia a procesos pedagógicos y didácticos en los que el diseño de los currículos, planes de estudio y syllabus está orientado al desarrollo de competencias ambientales (Mora, 2015).

3. Investigación ambiental: La Política Nacional de Investigación Ambiental (Ministerio de Ambiente y Desarrollo Sostenible, Departamento Nacional de Planeación y Colciencias, 2001, p. 6) la define como "aquella que se ocupa del estudio del entorno físico-biótico, de su relación con la estructura sociocultural, y de las dinámicas que tal relación conlleva".

4. EXTENSIÓN O PROYECCIÓN SOCIOAMBIENTAL: En la interacción e integración con el entorno, las IES identifican diversos problemas ambientales que la sociedad demanda, entre ellos, los relacionados con el ambiente, y a partir de ahí los articula en la búsqueda de una solución a través de la integración de la docencia y la investigación.

5. Gestión ambiental: Es un eje central en los procesos formativos de la institución, a través del cual se debe enseñar con el ejemplo, a partir de la intervención del contexto biofísico, desarrollando un conjunto de procesos enfocados a mitigar los impactos de las actividades de la institución. Para el presente estudio, se indagaron aspectos relacionados con la gestión propia del campus, por ejemplo: gestión de residuos, uso eficiente de recursos, movilidad sostenible, consumo responsable, entre otros.

\section{Herramientas de medición de la sustentabilidad aplicadas a las universidades}

La evaluación de la contribución de la educación superior a la sustentabilidad es llevada a cabo a través de diferentes herramientas. Con frecuencia se utiliza el envío de un cuestionario en línea y también se realiza la presentación de reportes de sustentabilidad o la participación en rankings. En relación con el Global Reporting Initiative (GRI, 2015), a pesar de que no exige un proceso independiente de verificación, sí se alienta a tenerlo en cuenta, pues afirma que esta iniciativa puede proporcionar tanto a los lectores del informe como a los gerentes internos una mayor confianza en la calidad de los datos de desempeño de la sustentabilidad, lo que hace más probable que se los utilice en la toma de decisiones.

En el ámbito de la educación superior, Fonseca et al. (2011) verificaron los informes de sostenibilidad publicados por 25 universidades de Canadá y encontraron que ellos tienen un valor limitado y son potencialmente engañosos como una herramienta para informar decisiones orientadas a la sostenibilidad. Por lo tanto, el proceso de verificación evidenció que la información publicada por las universidades no estaba cumpliendo con lo esperado.

Por su parte, en Perú, Cárdenas (2014) indagó sobre la incorporación de la perspectiva ambiental en 37 universidades. El proceso consistió en la aplicación de un cuestionario enviado a las IES. Posterior a la recepción de las respuestas, procedieron a la verificación de los documentos e información aportada en tres formas: análisis y confrontación de las evidencias que adjuntaban, revisión de sitios web y, en algunos casos, consultas directas a los responsables.

En un estudio realizado en España por CRUE (2010) se evaluaron las políticas universitarias de sostenibilidad en 31 universidades españolas. Para ello se envió un cuestionario virtual y posteriormente se realizaron entrevistas presenciales. El proceso de verificación estuvo enfocado en la indagación de la comprensión de las preguntas del cuestionario 
por parte de la persona que lo había diligenciado. El proceso permitió cambiar las respuestas, al mejorar la comprensión de la pregunta.

Por otra parte, el Greening Universities Toolkit concibe estrategias básicas necesarias para que las universidades puedan transformarse en instituciones verdes, con capacidad para abordar el cambio climático, aumentar la eficiencia de los recursos, mejorar la gestión de los ecosistemas y minimizar los residuos y la contaminación, centrándose en la planificación, diseño, desarrollo y gestión sostenible de los campus (UNEP, 2013). Adicionalmente se encuentra el Green Metric Ranking, el cual es una iniciativa de Universitas Indonesia (2015), cuyo resultados están basados en una puntuación numérica que permite realizar una clasificación a partir del compromiso ambiental de las universidades.

\section{Metodología}

La investigación planteada en este artículo tiene un alcance de tipo exploratorio, en cuanto se busca identificar aspectos de la institucionalización de la sustentabilidad en un grupo de universidades colombianas, un tema poco estudiado en el país. De acuerdo con Hernández, este tipo de estudios sirve para familiarizarse "con fenómenos relativamente desconocidos, obtener información sobre la posibilidad de llevar a cabo una investigación más completa respecto de un contexto particular" (2014, p. 91).

\section{Diseño de la herramienta y aplicación del cuestionario}

La herramienta se diseñó con las 114 preguntas propuestas en el proyecto de la Red de Indicadores de Sostenibilidad Universitaria (RISU), las cuales fueron convalidadas con el esfuerzo colectivo de un grupo importante de universidades latinoamericanas pertenecientes a la Alianza de Redes Iberoamericanas de Universidades por la Sustentabilidad y el Ambiente (ARIUSA) (Benayas et al., 2014). Para el pre- sente estudio, se eligieron 25 de las 114, que se identificaron como "básicas" para la identificación de aspectos de ambiente y sustentabilidad en las IES.

Por su parte, el proyecto RISU dividió el cuestionario en 11 temáticas: 1) Política de sostenibilidad; 2) Sensibilización y participación; 3) Responsabilidad socioambiental; 4) Docencia; 5) Investigación y transferencia; 6) Urbanismo y biodiversidad; 7) Energía; 8) Agua; 9) Movilidad; 10) Residuos, y 11) Contratación responsable. Para el presente estudio, se decidió simplificar el número de temáticas, pues se consideró que a partir de la dimensión 6 de RISU, correspondiente a "Urbanismo y biodiversidad" y hasta la 11, "Contratación responsable", se podrían agrupar bajo un solo ámbito, designado como "Gestión y ordenamiento ambiental del campus".

A partir de dicho análisis, y a través de una amplia revisión de estudios a nivel internacional, se definieron los siguientes cinco "ámbitos" de acción institucional: 1) gobierno y participación; 2) docencia y formación; 3) investigación y tecnología; 4) extensión o proyección; 5) gestión y ordenamiento.

\section{Selección de las preguntas}

Con el fin de obtener información básica y primordial de las universidades colombianas, a partir de la revisión de un grupo de seis expertos', se tomó la decisión de elegir cinco preguntas para cada uno de los cinco ámbitos, con un total de 25 que dio lugar a una nueva encuesta, que se considera básica para la evaluación del compromiso con la sustentabilidad de las IES.

El instrumento estuvo conformado por preguntas cerradas, con un espacio para la ampliación de la información, como también para reportar información

1 La mayoría de sus integrantes llevan más de una década trabajando con las universidades promoviendo la incorporación de los temas de ambiente y sustentabilidad y, de acuerdo con su experiencia, consideran en su conjunto han alcanzado un nivel medio en el cumplimiento de los indicadores utilizados en la encuesta. 
publicada en la web institucional u otro medio disponible para el público. El envío del instrumento se realizó a través de Google Formularios, invitando a las IES a diligenciarlo con información de carácter institucional. La encuesta fue respaldada por la Asociación Colombiana de Universidades (AsCuN), como también por la Red Ambiental de Universidades Sostenibles (RAUS) y la Red Colombiana de Formación Ambiental (RCFA), dos organizaciones que han venido acompañando los procesos de institucionalización del compromiso ambiental de la IES colombianas.

\section{Escala de análisis}

Para el análisis se estableció una escala de orden para cada uno de los ámbitos, como también para el total de las respuestas. Para cada ámbito se determinaron diferentes niveles de acuerdo con la respuesta proporcionada. Para este análisis no solo se tuvo en cuenta si la respuesta era positiva o negativa, sino que adicionalmente se analizó: 1) si la ampliación de la información era pertinente; 2) si la información está institucionalizada a partir de un documento formal tipo resolución o acuerdo, y, adicionalmente, 3 ) si se hace divulgación de estos a través de las páginas web de la universidad. La puntuación proporcionada a cada pregunta se explica a continuación:

1. Si la respuesta es negativa, puntuación: 0 .

2. Si la respuesta es positiva, puntuación: 1 .

3. Si se incluye una ampliación pertinente de la información, puntuación: 2.

4. Cita documento institucional relacionado con aprobación oficial, puntuación: 3

5. Divulga a través de su página web la información relacionada, puntuación: 4 .

De esta manera, la puntuación máxima por pregunta es de 4, para un total de 20 puntos por ámbito. A partir de la puntuación proporcionada, se estableció la siguiente escala de orden para cada ámbito: o-4 puntos: nivel muy bajo.

4,1-8 puntos: nivel bajo.

8,1-12 puntos: nivel medio.

12,1-16 puntos: nivel alto.

16,1-20 puntos: nivel muy alto.

A partir de la calificación para cada uno de los ámbitos, se estableció también una escala para el análisis del total de las 25 preguntas por universidad, la cual variaba entre o y 100. La mayor puntuación posible (100) se da a partir de la multiplicación de los 5 ámbitos, por la mayor puntuación en cada uno de ellos, la cual era de 20. La escala general usada se muestra a continuación:

$$
\begin{aligned}
& \text { 0-20,9 \%: muy bajo } \\
& \text { 21-40,9\%: bajo } \\
& \text { 41-60,9\%: medio } \\
& \text { 61-80,9\%: alto } \\
& \text { 81-100 \%: muy alto }
\end{aligned}
$$

Para el análisis de cada uno de los cuestionarios diligenciados, se socializó la metodología establecida con un grupo de seis expertos de cinco IES, realizando varios ejercicios de manera conjunta para unificar criterios y proponer un protocolo de análisis estandarizado. Posteriormente, cada uno proporcionó el puntaje por pregunta, de acuerdo con el mecanismo establecido. Después de esto, se tomó una muestra al azar de 20 universidades, para asegurarse de la uniformidad en la puntuación proporcionada por los seis expertos.

\section{Establecimiento de la muestra}

El Ministerio de Educación Nacional afirma que en 2014 el país contaba con un total 288 instituciones de educación superior, de las cuales solo 82 eran universidades. Dadas las condiciones de las universidades colombianas, en cuanto a la institucionaliza- 
ción del compromiso con la sustentabilidad, se establece un muestreo por conveniencia, una técnica no probabilística donde los sujetos son seleccionados por su accesibilidad para el investigador. Es decir, la encuesta fue diligenciada por las universidades que voluntariamente quisieron considerar su aporte. La encuesta fue respondida por 36 IES de diferentes regiones del país, reconocidas oficialmente como universidades, que corresponden al 43,9\% del total (82) y suministran una información significativa sobre la situación actual del proceso estudiado.

\section{Verificación y análisis del resultado}

Para la sistematización de la información se utilizó el método de tabulación automatizado, mediante el cual, con el apoyo de equipos electrónicos "se hace una concentración más rápida de resultados a través de la tabulación y clasificación de información. Los resultados se presentan así en forma más confiable, con mayor profundidad y en un menor tiempo" (Muñoz, 2011, p. 121). Este fue el caso de la utilización de Google Formularios, una herramienta que permite realizar la recopilación de forma automática y ordenada. Por otra parte, se utilizaron criterios de estadística descriptiva donde se interpretan los datos y se los presenta en gráficas, cuadros y resúmenes. Para las universidades que respondieron la encuesta, se realizó análisis de frecuencias, identificando la media para el puntaje total de las IES y para cada uno de los cinco ámbitos, teniendo en cuenta puntajes mínimos y máximos.

\section{Resultados}

La Figura 1 presenta los puntajes generales obtenidos por cada una de las 36 universidades, en orden ascendente. Como se puede apreciar, el menor puntaje obtenido fue de 13 y el máximo de 81 , con un rango de 68 puntos, lo que muestra una importante dispersión de los datos. El promedio general fue de 41 puntos, que es el puntaje mínimo de la categoría que se calificó como nivel medio, y se estableció que 21 de 36 universidades están en el nivel bajo y muy bajo.
De acuerdo con la Figura 1, se puede colegir el nivel en el que se encuentran las 36 universidades. Casi la mitad de ellas se ubican en el nivel bajo, con puntajes entre 21 y 40, y 9 alcanzan el nivel medio, al obtener entre 41 y 60 puntos; 5 presentan un nivel alto y solo 1 obtiene un nivel muy alto, mientras que 4 universidades tienen un nivel muy bajo. Los resultados de la encuesta coinciden entonces con el supuesto inicial: que el nivel de institucionalización del compromiso con el ambiente y la sustentabilidad en las IES en Colombia es incipiente.

Comparado con un estudio exploratorio previo, de los mismos proponentes de este documento (Sáenz et al., 2017) sin realizar verificación, se encontró que los datos están desplazados del orden en las categorías, de modo que los que en el estudio anterior aparecían como altos pasan a estar en el nivel medio y algunos de los que estaban en nivel medio pasan al bajo, cuando a las respuestas positivas se les aplican los criterios verificación.

Otro aspecto que interesa conocer es el avance de la institucionalización del compromiso en cada uno de los 5 ámbitos definidos, para agrupar los 25 indicadores básicos. Los resultados que agrupan los 5 ámbitos se presentan en la Figura 2.

Para cada uno de los 5 ámbitos de acción se seleccionaron 5 preguntas básicas, a las cuales se les asignó un puntaje máximo de 4, si cumplían con todos los criterios de verificación. Consecuentemente, el puntaje más alto posible en cada ámbito fue de 20. En la Figura 2 se aprecia que, de los 5 ámbitos de acción universitaria para la institucionalización de la universidad, 4 se encuentran entre 7 y 8, es decir, en el rango de bajo, y solo 1 ámbito, el de docencia y formación ambiental, está en el rango medio entre 9 y 12. Este resultado también es coherente con el supuesto de partida, donde se afirma que en las universidades existe una incipiente ambientalización, por lo cual se esperaba que el promedio de las universidades se ubicara en la categoría de bajo. 
Figura 1. Puntaje general para cada universidad

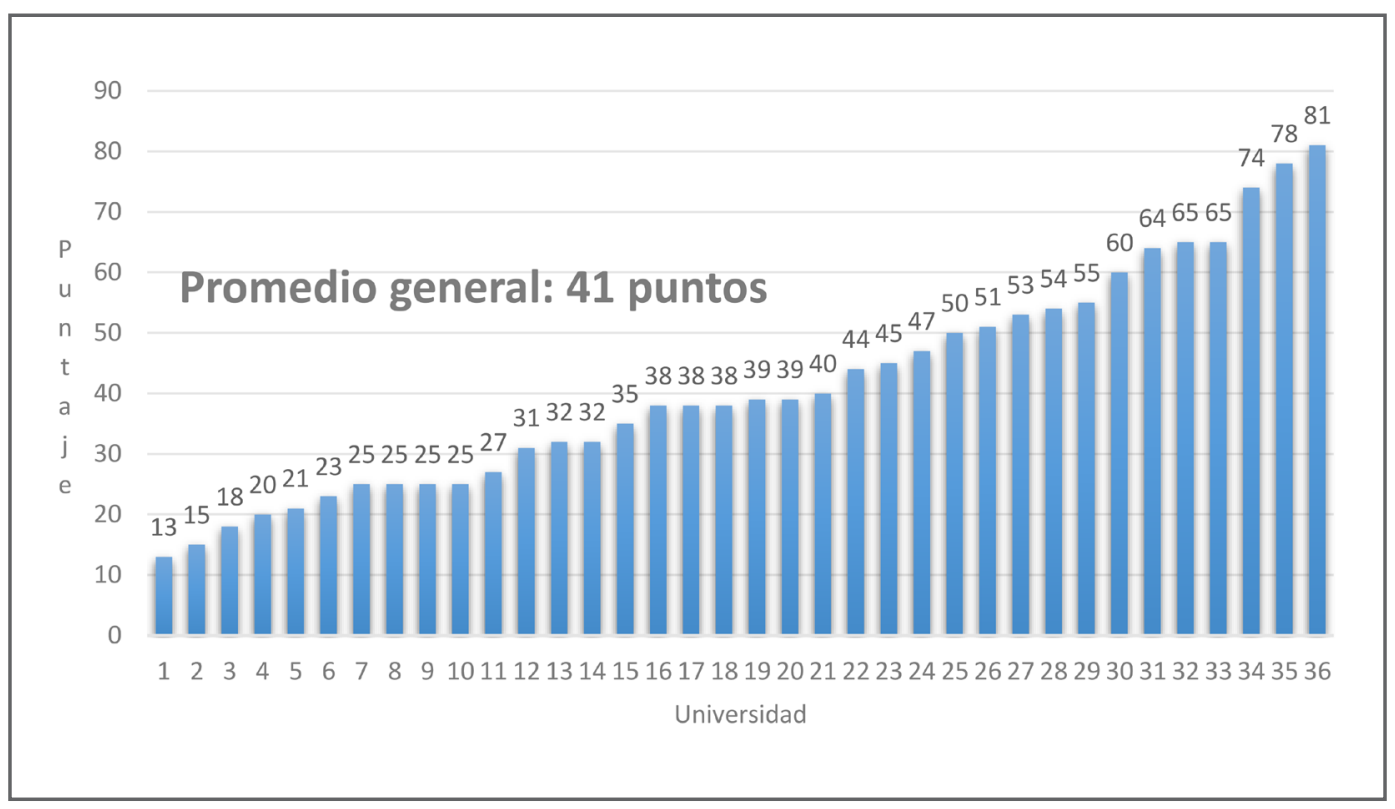

Fuente: Elaboración propia.

\section{Figura 2. Puntajes promedio en los 5 ámbitos de acción universitaria en Colombia, 2014}

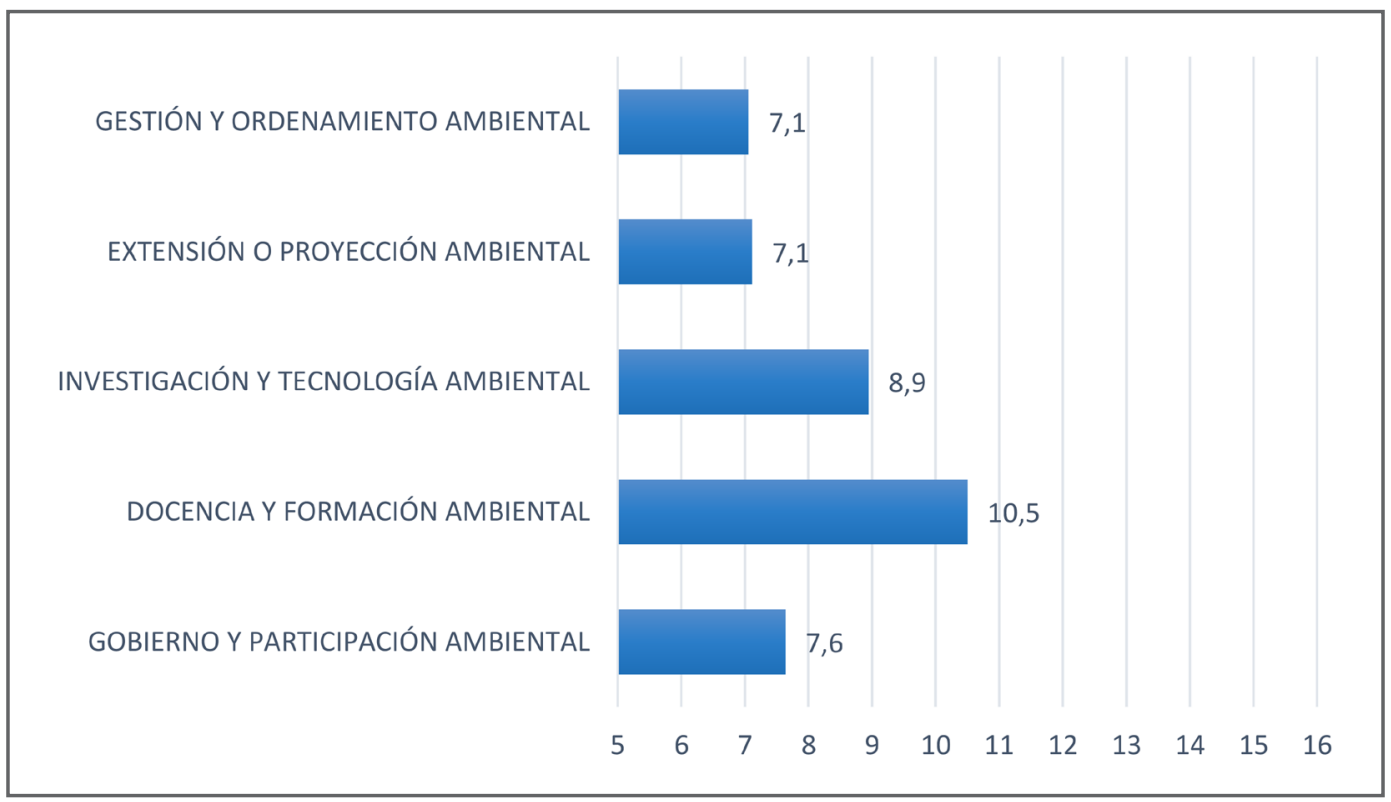

Fuente: Elaboración propia. 
De igual manera, es coherente el orden en que se presentan en la Figura 2 los niveles de institucionalización del compromiso con el ambiente y la sustentabilidad por ámbito. Este orden corresponde en gran medida al conocimiento que se tiene del proceso histórico de incorporación de la dimensión ambiental en los diferentes ámbitos de acción de las IES. En general, este comenzó en Colombia desde finales de la década del setenta y desde un principio el mayor énfasis se hizo en la creación de nuevos programas ambientales y en la incorporación de temas afines en todos los programas de formación profesional. Por lo tanto, no sorprende que el ámbito de acción con mayor puntaje (de 10,5) sea el de docencia y formación. Un buen número de universidades colombianas tienen programas de pregrado y posgrado especializados en temas de ambiente y sustentabilidad y algunos avanzan en la incorporación de estos temas en los demás programas.

El segundo ámbito de acción con mayor puntaje es el de investigación, con un valor de 8,9. Este es también un resultado que coincide con lo esperado a partir del conocimiento que se tiene del proceso histórico, pues desde la década del noventa la investigación ambiental es el otro campo de acción que más se ha promovido en las instituciones de educación superior, por parte de las agencias gubernamentales de ciencia, tecnología y educación en Colombia, y a partir de 2001 los aspectos ambientales hacen parte de los compromisos éticos en todo proyecto de investigación presentado ante Colciencias y soportado en la política nacional de investigación ambiental.

De acuerdo con el conocimiento previo, se esperaba que el tercer puntaje correspondiera al ámbito de extensión o proyección social. Las mismas agencias estatales han promovido también el trabajo de las universidades sobre problemáticas ambientales y de sustentabilidad en relación directa con las comunidades, las empresas y los gobiernos locales. Sin embargo, este ámbito de acción aparece en el cuarto lugar con puntaje de 7,1, por razones que se analizarán más adelante.
La misma situación se refleja en el ámbito de gestión y ordenamiento ambiental con puntaje de 7,1, en el cual se esperaría también que hubiese mayor avance; sin embargo, existen aún grandes retos para continuar avanzando en la gestión ambiental y garantizando un desarrollo más armónico del campus.

Por encima de lo esperado, el ámbito de gobierno y participación aparece como el tercero, con puntaje de 7,6. Este resultado puede reflejar el énfasis que se viene haciendo en los últimos años sobre institucionalización del compromiso con la sustentabilidad, a través de la formulación de política ambiental universitaria, la formalización de la acción por el ambiente y la sustentabilidad a través de planes y programas y la participación de la comunidad universitaria.

Por otra parte, el análisis comparativo por ámbito entre Fase I y Fase II es muy importante, cuando se analiza el comportamiento de los datos sin verificación y con verificación. Como se explicó en la metodología, un primer momento del análisis se basó en las respuestas obtenidas en cada pregunta a partir de los descriptores "SÍ" o "NO", pero en la segunda fase se hizo lectura de los datos aportados en el descriptor “Ampliación de la información” “"Página web relacionada". En la ampliación se analizaba además la pertinencia de la información y si respondía a información soportada por documentos oficiales de orden institucional.

En la Figura 3 se puede observar la variación de los datos, los cuales van en descenso del desempeño ambiental con verificación y sin verificación. Uno de los aspectos analizados para que se presente este fenómeno es, por ejemplo, el desarrollo de acciones aisladas con deficientes procesos de sistematización y documentación de sus avances. En ocasiones se cuenta con la información, pero no se potencian los medios de divulgación, pues se afirma que, al ser información institucional, no es susceptible de divulgación. Otro aspecto no menos importante es que en algunos casos la encuesta fue contestada por 
una sola persona y no por un equipo, como pedían las instrucciones, lo cual refleja ausencia de información que debe ser compilada con actores tanto académicos como administrativos.

Profundizando un poco más sobre la ampliación de la información aportada, se han identificado algunas tendencias de las Universidades. Por ejemplo, en el "Ámbito participación y política ambiental", de las 36 Universidades que respondieron la encuesta solo 8 dicen no tener una política ambiental; sin embargo 3 señalan que se encuentran en el proceso de aprobarla. Dentro de las 28 que responden que sí tienen política, solamente hay 4 que están en proceso de formalización, lo que permite evidenciar que existe un avance progresivo en la formalización de su política ambiental en las universidades colombianas.

Una afirmación recurrente en esta pregunta es el hecho de que 6 universidades, a pesar de indicar que no tienen una política exclusiva de ambiente, indican que sí tienen integrado el tema a través del PEI, la misión y visión de la Universidad; adicionalmente, en algunos casos lo ambiental aparece dentro de otras políticas, como pueden ser las de calidad o seguridad y salud en el trabajo.

Adicionalmente, aunque no es el factor dominante, 7 universidades en su ampliación de la información afirman tener una resolución o documento formal a través del cual se institucionaliza la política ambiental. Sin embargo, al momento de la aplicación de la encuesta no tenían dicho documento de respaldo.

En este mismo ámbito, respecto de la pregunta 3: "La organización de la acción por el ambiente y la sustentabilidad en su universidad corresponde: a) un conjunto de acciones o agenda, b) un Plan de Acción Ambiental y c) un Sistema Ambiental Institucional"-, hubo una pequeña excepción a la regla de

Figura 3. Análisis por ámbitos comparando Fases 1 y 2

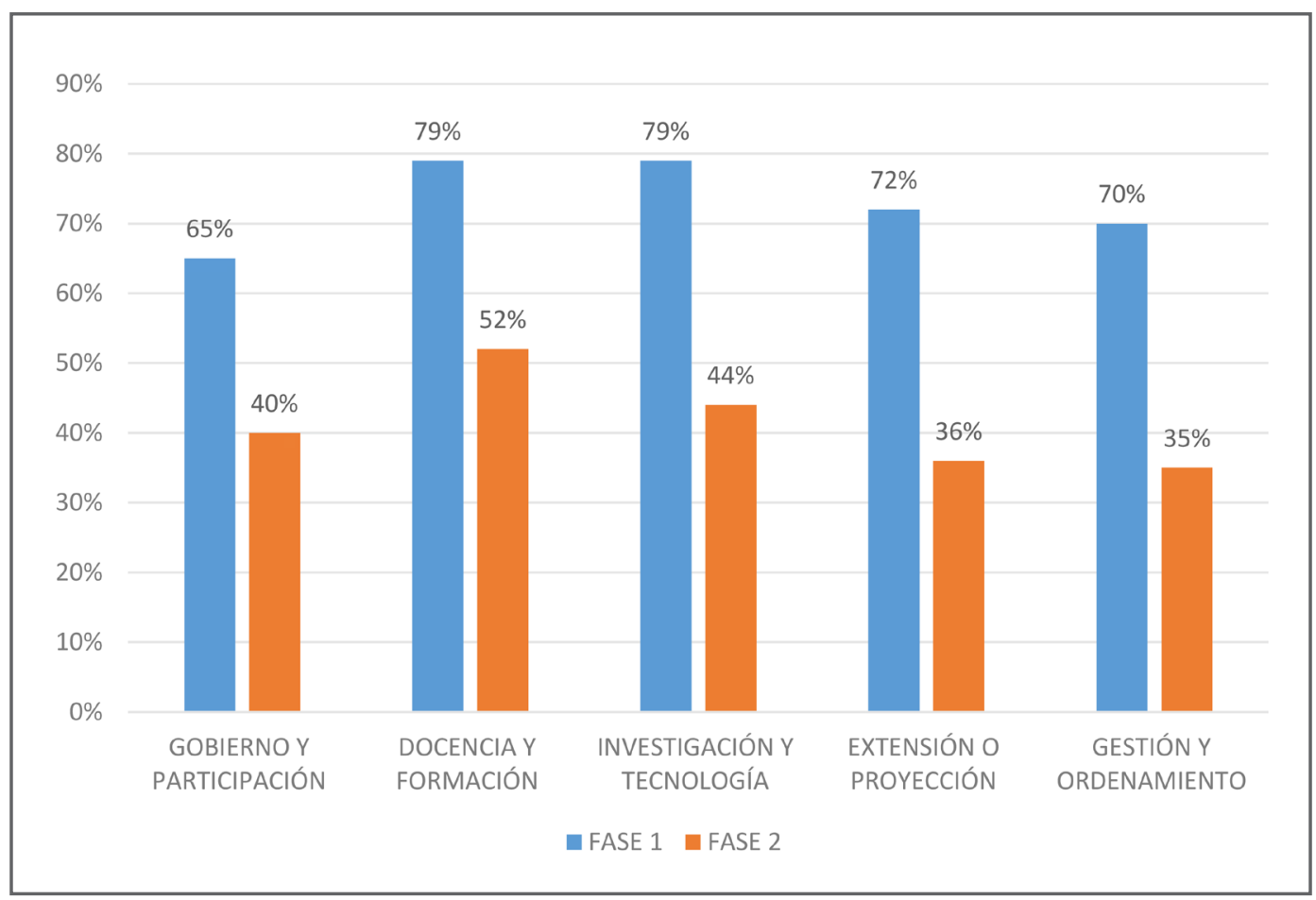

Fuente: Elaboración propia. 
evaluación, pues, como se puede observar, había opciones adicionales a la respuesta Sí o NO (Sáenz et al., 2017). Entonces, dada la importancia de la pregunta para analizar la "institucionalización del compromiso con la sustentabilidad", se dio prioridad a las respuestas b) o c), ya que daban cuenta de un nivel de organización más complejo de las acciones. De este modo dichas opciones proporcionaban una mayor puntuación que tener un conjunto de acciones.

Los resultados de la Fase 1 demuestran que, de las 36 universidades, 25 señalaron que su acción por la sustentabilidad se debía a un Sistema Ambiental Institucional o un Plan de Acción Ambiental, y 11 universidades indicaron que solamente tenían un conjunto de acciones ambientales o agenda. En el proceso de verificación en la Fase 2, se determinó que, entre las 25 universidades mencionadas arriba, 17 indican tener un Sistema Ambiental Institucional y 8 un Plan de Acción Ambiental, lo cual muestra que las universidades reconocen diferencias en la concepción del Sistema Ambiental y del Plan de Acción.

A diferencia de lo que se esperaba, se determinó que, de las 17 IES que decían tener SAI, solo 3 demostraron tener un nivel alto de institucionalización del compromiso con la sustentabilidad y 5 un nivel medio. Las 7 restantes instituciones obtuvieron un nivel bajo y 2 muy bajo, lo que lleva a concluir que es necesario reconceptualizar con las universidades la categoría de SAI, dado el interés que representa para la investigación el hecho de trascender desde allí hacia estadios más altos de su compromiso.

Al analizar la relación de la pregunta 1 (existencia de una política ambiental universitaria) y la 3 (la organización de la acción por la sustentabilidad) respecto al desempeño final de las Universidades, se pueden identificar varios hallazgos (Figura 4).

\section{Figura 4. Relación de la organización de la acción por la sustentabilidad y existencia de una política ambiental institucional}

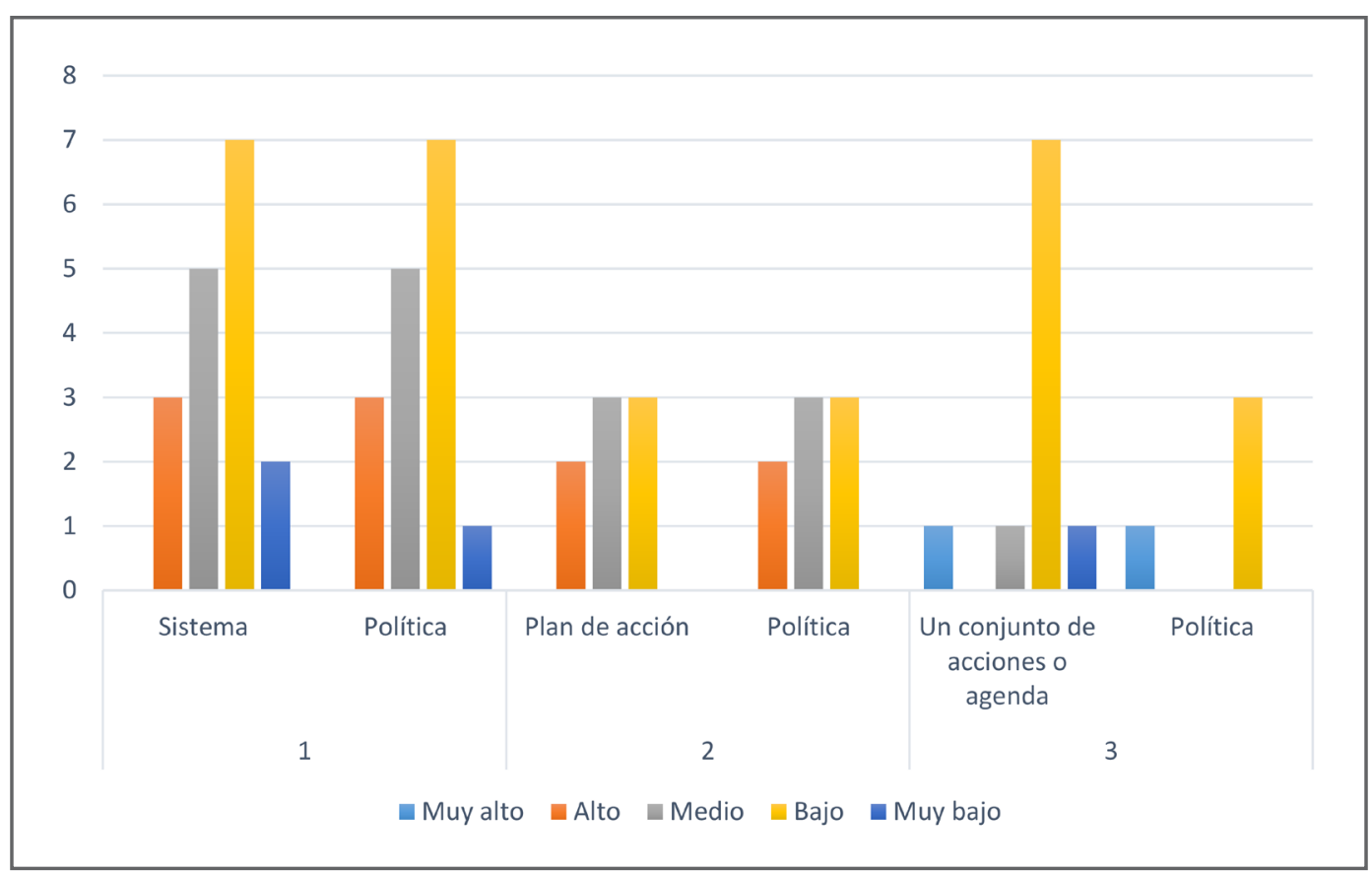

Fuente: Elaboración propia. 
En la Figura 4 se puede observar que un grupo de 7 universidades obtuvo bajo y 2 muy bajo en la valoración, aun a pesar de haber indicado la existencia de un sistema ambiental institucional y una política ambiental universitaria. Solo una universidad se ubicó en nivel muy alto; esta institución indica tener política ambiental, pero estar trabajando solo un conjunto de acciones o agenda, que sumadas pueden dar cuenta de un proceso sistemático que cuenta con evidencias.

Por el contrario, 9 de las universidades con puntajes más bajos señalaron tener sistema, pero no política ambiental. Este hallazgo es importante, porque parece demostrar que, entre más avances demuestra una universidad en el proceso, más conciencia tienen sus gestores sobre la perspectiva sistémica, razón por la cual se es más discreto en su denominación, y que, entre menos avances, su forma de denominar el proceso indica desconocimiento de la categoría sistema ambiental por parte de quienes contestaron el instrumento.

Para el análisis del ámbito de "Docencia y formación ambiental”, según la forma de ponderación de la información, que propone la Tabla 2, entre o y 4 , se puede decir que en promedio las 5 preguntas de este ámbito se encuentran en un estado intermedio, donde hay respuestas positivas que incluyen ampliación de la información, pero en su mayoría no se presentan canales de verificación oficial ( $F i$ gura 5). Las preguntas donde más se evidencia la verificación son la 9 y la 10, las cuales adicionalmente son las de mayor puntuación, pues, cuando las IES cuentan con programas formales como pregrados y posgrados, siempre se encuentran sus documentos en las páginas web de las instituciones. Es evidente entonces el crecimiento de programas de formación en temas ambientales, lo cual no solo sucede en Colombia. En Inglaterra, por ejemplo, desde la década del setenta ya se ofrecían grados académicos en Ciencias Ambientales y, posteriormente, en 1987, 15 universidades inglesas ya contaban con dichos programas (Leal-Filho, 1999).
En la Figura 5 se observa que solo 15 de 36 universidades, es decir, el 41,6\%, están por debajo de 9 puntos y se ubican en bajo o muy bajo; 10 de 36 universidades (27,8\%) se encuentran en el nivel medio; y 11 de 36 universidades (30,6\%) alcanzan a estar entre alto y muy alto.

El ámbito de "Investigación ambiental", como se señaló anteriormente, es el segundo en el desempeño de las universidades, con un promedio de 8,9, lo que lo deja en un nivel medio. Así, por ejemplo, las ideas más recurrentes cuando se pregunta por la investigación dentro del campus de las IES son: se realiza a través de semilleros y trabajos de aula, a través de trabajos de grado; se aplica a planes de manejo ambiental o programas dentro del SGA, o se hace a través de proyectos de investigación propiamente dichos. Sin embrago, dentro de las universidades que afirmaron tener estrategias para incentivar el uso del campus de la universidad en la realización de investigaciones sobre temas ambientales, se encontró que la idea más recurrente, con un total de 6 universidades, era la investigación aplicada a planes de manejo ambiental o programas dentro del sistema de gestión ambiental.

A pesar de que este ámbito ocupó el segundo lugar, tal como lo afirman Kanashiro et al. (2010), es necesario tener en cuenta que, en términos generales, hay aún carencia de conocimientos y prácticas en gestión ambiental que ayuden a enfrentar la crisis socioambiental. Dichos conocimientos solo serán eficientemente generados por medio de estudios, investigaciones y capacitación de profesores, investigadores y profesionales, formados de forma multidisciplinaria e interdisciplinaria.

Respecto al ámbito de "Extensión y proyección socioambiental", el promedio de las 36 universidades fue de 7,1. En relación con la existencia de programas permanentes de vinculación con el sector empresarial del entorno en materia de ambiente o sustentabilidad, 22 universidades responden que sí, de las cuales 8 no amplían la información y solo 
Figura 5. Preguntas del ámbito de docencia y formación por universidad

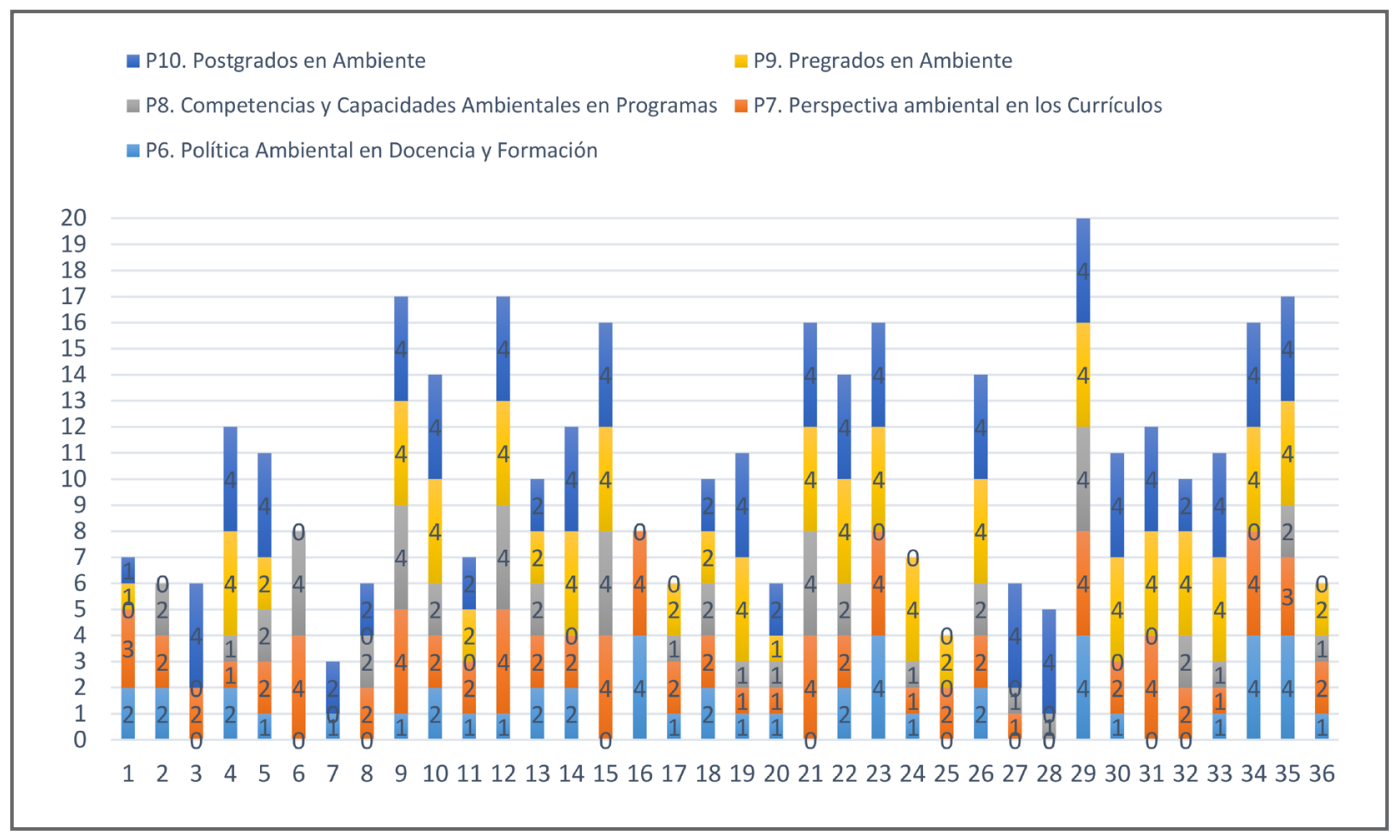

Fuente: Elaboración propia.

7 muestran el sitio web. Las 14 restantes responden que NO tienen estos programas. Al ampliar la información, se refieren a prácticas empresariales o sociales de los estudiantes, convenios con empresas y exigencia a los proveedores de la universidad.

Algo semejante sucede con los programas permanentes de intervención para contribuir a la solución de problemas ambientales o de sustentabilidad en comunidades, donde 13 universidades responden NO tener estos programas y 23 responden que Sí cuentan con ellos. Entre las acciones señaladas están las capacitaciones a las comunidades en temas ambientales, programa de paz y competitividad, prácticas sociales y empresariales, proyectos de recuperación y acciones de consultoría. Por otra parte, de las 36 universidades, 32 responden afirmativamente acerca de la participación en redes. De estas, 11 no amplían información y, de las 21 restantes, 9 pertenecen a RCFA y 5 a RAUS. Las demás indican que pertenecen a otras redes, varias de ellas internacionales.
En el ámbito de "Gestión y ordenamiento ambiental", el promedio de las 36 universidades fue de 7,1 , es decir, de bajo desempeño; por tanto, este será uno de los ámbitos en los que deberán avanzar con mayor compromiso las universidades colombianas. Como para este ámbito el promedio obtenido por cada pregunta oscila entre 1 y 1,9 , y de acuerdo con las escalas establecidas, se puede concluir que las universidades en general desarrollan actividades de gestión ambiental que no incluyen un proceso de seguimiento. Sin embargo, las universidades destacan algunas acciones que se presentan a continuación.

En lo relacionado con la existencia de un plan específico, eje estratégico o línea de acción que incluya aspectos de ahorro en agua, sanidad para consumo humano, riego y gestión de aguas residuales (Benayas et al., 2014), de las 36 universidades 25 indican contar con un plan o acciones asociadas al mismo. De estas, 22 ampliaron información, pero solo 6 tienen información relacionada en la web y 6 indican tener un programa oficialmente de ahorro y uso 
racional del agua; otras mencionan adelantar algunas estrategias como: implementación de sistemas ahorradores en lavamanos y sanitarios; aprovechamiento de aguas lluvias; sustitución de insumos de limpieza por otros ecológicos, para evitar la contaminación del agua; sensibilización sobre el consumo de agua embotellada; desarrollo de proyectos en las cuencas del territorio; mantenimiento preventivo de instalaciones hidrosanitarias; seguimiento a partir de indicadores de consumo de agua; y planta de tratamiento de aguas residuales.

Respecto a la existencia de un plan específico, eje estratégico o línea de acción que incluya aspectos relacionados con el tema de energía, 22 universidades respondieron positivamente y 20 de ellas ampliaron la información, pero solo 9 reportan tenerla publicada en la página web; mientras que 6 instituciones indican tener proyectos o programas encaminados al uso eficiente y racional de la energía, y las demás enuncian implementar algunas estrategias: reconversión tecnológica de luminarias a tipo led; seguimiento a los consumos de energía; automatización de los edificios. Solo 1 universidad menciona tener un grupo de investigación sobre el uso eficiente de la energía. La instalación de energías limpias, como paneles solares o aerogeneradores, parece aún débil en las universidades colombianas.

En cuanto al manejo adecuado de residuos, 31 universidades mencionan contar con un "plan específico, eje estratégico o línea de acción para la minimización, recogida selectiva y manejo adecuado de los residuos"; de estas, 22 ampliaron información y 12 indican tenerla publicada en página web. Este componente ha mostrado mayor gestión a lo largo de la historia de las universidades, lo cual se confirma también en el presente diagnóstico durante la fase de verificación, donde 15 universidades señalan tener un "Plan de manejo de residuos sólidos" propiamente dicho. Las demás reportan la implementación de algunas de las siguientes estrategias: manejo de residuos peligrosos con gestores externos; planta de compostaje, con selección de residuos só- lidos en la fuente, para lo cual han dispuesto puntos ecológicos y centros de acopio; recuperación de residuos potencialmente reciclables; recolección de residuos posconsumo: pilas, luminarias de mercurio, llantas, celulares; campañas para promover el reúso del papel; ecopuntos con sistemas de estímulos; recolección de tapas, entre otros.

Sobre trabajo en ambiente y biodiversidad, 22 instituciones responden afirmativamente, pero solamente 7 de ellas indican contar con un plan o programa específico y las demás citan algunas de las estrategias que vienen desarrollando: inventario de especies de fauna y flora, acciones de ornato y paisajismo, proyectos de cuidado y conservación de la biodiversidad, entre otros.

Finalmente, frente a la pregunta sobre planificación urbanística, 26 IES responden positivamente y 18 amplían información pertinente, pero solo 4 indican tener publicado en la página web la información. En este ítem no se enuncian realmente las estrategias, como sucedió con los demás aspectos ambientales analizados.

\section{Conclusiones}

Las universidades colombianas están avanzando en el compromiso con la sustentabilidad; sin embargo, se confirmó el postulado inicial de que aún se encuentran en un nivel incipiente o bajo. Ello se comprueba en el resultado promedio por ámbitos, con 4 en el rango bajo y solo 1 , el de docencia y formación ambiental, en el rango medio. Dichos resultados tienden a desmejorar cuando se avanza a los procesos de verificación. Por lo anterior, las universidades deben reforzar sus procesos de documentación y divulgación.

Es necesario que las IES valoren la perspectiva sistémica a la hora de adelantar procesos de inclusión de los temas de ambiente y sustentabilidad. Para ello será necesario reforzar esta categoría de análisis a través de procesos de capacitación y producción académica de estudios como este que de- 
muestren sus bondades. Convertir el proceso en un sistema ambiental y a la vez desarrollarlo a través de la investigación permanente puede ser una estrategia de mediano y largo plazo que induzca al seguimiento y socialización permanente de sus avances.

Sin duda un proceso de estas características requiere de la decisión efectiva de las directivas, quienes tienen a cargo la toma de decisiones acerca de la creación de los departamentos de formación y gestión ambiental, la asignación de presupuestos, la pronación de compras sustentables, la transformación hacia infraestructuras sostenibles, la posibilidad de convenios con el sector el Estado y el sector productivo para el desarrollo de proyectos de investigación y proyección social que permitan impactar positivamente las comunidades.

\section{Referencias}

Alonso-Almeida, M., Marimon, F., Casani, F. y Rodriguez-Pomeda, J. (2015). Diffusion of sustainability reporting in universities: Current situation and future perspectives. Journal of Cleaner Production, 106, 144-154. http://dx.doi.org/10.1016/j.jclepro.2014.02.008

Amérigo, M., García, J., Côrtes, P. (2017). Análisis de actitudes y conductas pro-ambientales: un estudio exploratorio con una muestra de estudiantes universitarios brasileños. Ambiente \& Sociedade, 20(3), 1-20. http:// www.scielo.br/pdf/asoc/v20n3/es_1809-4422-asoc-20-03-00001.pdf

Benayas J., Alba D., Justel A. (2014). Proyecto RISU. Definición de indicadores para la evaluación de las políticas de sustentabilidad en universidades latinoamericanas. https://digi.usac.edu.gt/edigi/digital-o2.html

Cárdenas, J. M. (2014). Incorporación de la perspectiva ambiental en las universidades peruanas. Reporte sobre el compromiso con la sustentabilidad de las universidades. http://www.minam.gob.pe/educacion/wpcontent/uploads/sites/20/2015/og/Informe-Inclusi\%C3\%B3n-de-lo-ambiental-en-las-universidades_2O-DEJULIO.pdf

Ceulemans, K., Lozano, R. y Alonso-Almeida, M. M. (2015). Sustainability reporting in higher education: Interconnecting the reporting process and organizational change management for sustainability. Sustainability, 7(7), 8881-8903. https://doi.org/10.3390/su7078881

CRUE (2010). Evaluación de las políticas universitarias de sostenibilidad como facilitadoras para el desarrollo de los campus de excelencia internacional. CADEP. http://www.crue.org/Documentos\%2ocompartidos/ Estudios\%20e\%2olnformes/21.CADEP2011finalbaja.pdf

Fonseca, A., Macdonald, A., Dandy, E., Valenti, P. (2011). The state of sustainability reporting at Canadian universities. International Journal of Sustainability in Higher Education, 12(1), 22-40. http://dx.doi. org/10.1108/14676371111098285

Global Reporting Initiative (2015). G4. Guía para la elaboración de memorias de sostenibilidad. Principios y contenidos básicos. http://www.mas-business.com/docs/Spanish-G4.pdf

González-Gaudiano, E., Meira-Cartea, P. y Martínez-Fernández, C. (2015). Sustentabilidad y Universidad: retos, ritos y posibles rutas. Revista de la Educación Superior, 44(175), 69-93. http://www.scielo.org.mx/scielo. php?script=sci_arttext\&pid=S0185-27602015000300004\&lng=es\&tlng=es 
Hernández-Sampieri, R. (2014). Metodología de la investigación (6 ed.). McGraw-Hill. https://www.uca. ac.cr/wp-content/uploads/2017/10/Investigacion.pdf

Holguín, M. T. (2017). Inclusión de la dimensión ambiental desde la perspectiva sistémica en la educación superior. "Estudio de caso de la Universidad Libre-sede principal-como referente para un modelo institucional". Universidad Libre. http://www.unilibre.edu.co/bogota/pdfs/2017/siaulmt.pdf

Kanashiro- Uehara, T., Prol-Otero G., Andrade-Martins E., Philippi, A. y Mantovani W. (2010). Pesquisas em gestão ambiental: análise de sua evolução na Universidade de São Paulo. Ambiente \& Sociedade. 13(1), 165-185. http://www.scielo.br/pdf/asoc/v13n1/v13n1a11.pdf

Koester, R. J., Eflin, J. y Vann, J. (2006). Greening of the campus: a whole-systems approach. Journal of Cleaner Production, 14(9-11), 769-779. https://www.researchgate.net/publication/229392807_Greening_of_ the_campus_a_whole-systems_approach

Leal-FilhoW. (1999). Meio ambiente:um tema de valor estratégico para a universidade brasileira.Ambiente \& Sociedade, 2(5),191-201. http://www.scielo.br/scielo.php?script=sci_arttext\&pid=S1414-753X1999000200013

Lozano R. (2006). Incorporation and institutionalization of SD into universities: breaking through barriers to change. Journal of Cleaner Production, 14(9), 787-796. https://doi.org/10.1016/j.jclepro.2005.12.010

Luhmann, N. (1990). Sociedad y sistema: la ambición de la teoría. Paidós.

Lukman, R., Glavič, P. (2006). What are the key elements of a sustainable university? Clean Technol Environ Policy, 9(2), 103-114. http://dx.doi.org/10.1007/s10098-006-0070-7

Ministerio de Ambiente y Desarrollo Sostenible, Departamento Nacional de Planeación y Colciencias (2001). Política Nacional de Investigación Ambiental. https://redjusticiaambientalcolombia.files.wordpress. com/2012/og/politica-nacional-de-investigacic3b3n-ambiental2.pdf

Ministerio de Educación Nacional y Ministerio de Ambiente (2002). Política Nacional de Educación Ambiental. http://cmap.upb.edu.co/rid=1195259861703_152904399_919/politi-ca_educacion_amb.pdf

Ministerio de Educación Nacional (2014). Estadísticas de Educación Superior. https://www.mineducacion.gov.co

Mora, W. (2015). Introducción a la construcción de un sistema ambiental para la Universidad Distrital. Documento de Trabajo.

Muñoz, C. (2011). Cómo elaborary asesorar una investigación de tesis. Pearson. http://www.indesgua.org. gt/wp-content/uploads/2016/o8/Carlos-Mu\%C3\%B10z-Razo-Como-elaborar-y-asesorar-una-investigacionde-tesis-2Edicion.pdf

PNUMA (2014). Decisión del XIX Foro de Ministros de Medio Ambiente para América Latina y el Caribe sobre Educación Ambiental para el Desarrollo Sostenible. Programa de las Naciones Unidas para el Medio Ambiente.

Román, Y. (2016). Sistema ambiental universitario: Modelo integrado de gestión para la inclusión de la dimensión ambiental y urbana en la educación superior. Universidad Piloto de Colombia. 
Sáenz, O., Plata, A. M., Holguín, M. T., Mora, W., Blanco-Portela, N. (2017). Institucionalización del compromiso ambiental de las universidades colombianas. Civilizar Ciencias Sociales y Humanas, 17(33), 189-207. http:// revistas.usergioarboleda.edu.co/index.php/ccsh/article/view/908/776

Sterling, S. (2004). Higher education, sustainability, and the role of systemic learning. En P. B. Corcoran y A. Wals (eds.), Higher education and the challenge of sustainability (pp. 49-70). Kluwer.

UNEP - United Nations Environment Programme (2013). Greening universities toolkit. Transforming universities into green and sustainable campuses: A toolkit for implementers. http://wedocs.unep.org/bitstream/ handle/20.500.11822/11964/Greening\%20University\%20Toolkit\%20V2.o.pdf?sequence=1\&isAllowed=y

Universidad Libre (2016). Sistema Ambiental Académico, Administrativo de la Universidad Libre - sIAUL. Informe de balance general 2010-2016. http://www.unilibre.edu.co/bogota/pdfs/2017/mt303a.pdf

Universitas Indonesia (2015). UI Green Metric. World University Rankings UI. http://greenmetric.ui.ac.id/

Vallaeys, F., De la Cruz, C.y Sasia, P. (2009). Responsabilidad social universitaria. Manual de primeros pasos. McGraw-Hill.

Wals, A. (2014). Sustainability in higher education in the context of the UN DESD: a review of learning and institutionalization processes. Journal of Cleaner Production, 62, 8-15. https://doi.org/10.1016/j.jclepro.2013.06.007 